\title{
SECONDRAY STRUCTURE AND SEQUENCEOFITS2-rDNA OF THE EGYPTIAN MALARIA VECTORANOPHELES PHAROENSIS (THEOBALD) \\ By
}

\author{
NAHLA M. WASSIM \\ Molecular Biology Unit, Faculty of Science, Suez University, Egypt
}

\begin{abstract}
Out of the twelve Anophelines present in Egypt, only five species known to be malaria vectors. Anopheles (An.) pharoensis proved to be the important vector all over Egypt, especially in the Delta. Anopheles sergenti proved to be the primary vector in the Oases of the Western Desert, An. multicolor in Faiyoum, An. stephensi in the Red Sea Coast, and An. superpictus in Sinai. Genomic DNA was isolated from single adult mosquito of An. pharoensis (Sahel Sudanese form), PCR was performed to amplify ITS2 region of rDNA using specific primers for 5.8S and 28S rDNA genes. The amplicons were purified, directly sequenced and aligned to the sequence of the same region of An. gambiae, using clustalw2. The length of ITS2-rDNA of $A n$. pharoensis was $411 \mathrm{bp}$. The GC content of the ITS2 reported $53 \%$ is consistent with spacer base composition in Anopheles species. The similarity between the two species was $52 \%$ and genetic distance was 0.46 . Variable simple sequence repeats (SSRs) are found at low frequency. The secondary structure of rDNA-ITS2was predicted by MFOLD and was -192; 60 to-195.32 kilocalories/mole.
\end{abstract}

Key words: Anopheles Mosquitoes, Genetic Variations, ITS2-rDNA, Egypt

\section{Introduction}

Anopheles pharoensis has been divided into at least two species which are widespread in Africa (Miles et al, 1983). The form (or species) found in East and South of Africa is zoophilic and does not act as a vector. The Sahel Sudanese form that covers an area extending from the Atlantic Coast to the Valley of the Nile and to the Middle East displays mixed, opportunistic behavior (Zahar, 1989; Mouchet et al, 2008). Out of twelve Anopheline mosquitoes present in Egypt, only five species are known to be malaria vectors. An. pharoensis proved to be the important vector all over Egypt, especially in the Delta (Zahar, 1975). An sergenti proved to be the primary vector in the Oases of the Western desert, while An. multicolor, An. stephensi and An. superpictus plays a predominant role in Al-Faiyoum, the red sea coast and in the Sinai respectively. Also, they are known to be malaria vectors in other countries.

Despite all the official efforts, malaria is still consider one of the health problems may threatening public health in Egypt (El Said and Farid, 1982; El Said et al, 1983; Mikhail et al, 2009). An evaluation of the clinical and parasitic status of malaria as a cause of fever among patients admitted to the Almaza Military fever hospital in Cairo only revealed the presence of thirty six malarial cases (El Bahnasawy et al, 2010). Besides, El Bahnasawy et al. (2011) recorded An. multicolor, An. sergentii, and An. algeriensis in Toshka Project, and added that $A$. sergentii is a malaria-vector and $A$. multicolor is a suspected vector. Consequently, the endemicity of the chloro-quine resistant Plasmodium falciparum on the Egyptian-Sudanese border pave the way for the malignant malaria trans-mission particularly among the travelers returning back from Sudan. 
The mosquito genus Anopheles (443 formally named species) contains all the vectors of human malaria parasites. Because many of the primary vectors belong to cryptic species complexes, it is necessary to have accurate phylogenetic reconstructions and species diagnostic tools. The evolution, molecular biology and biochemistry of rDNA have been the subject of intense study since it was characterized. The rDNA is a multicopy gene family that exists as one or more tandem arrays of many transcriptional units per cell, where concerted evolution rapidly spreads mutations to all members of the gene family, even if arrays are located on the different chromosomes (Dover, 1982; Gerbi, 1985; Tautz et al, 1988). Anopheles has the least amount of the repetitive DNA of any mosquitoes analyzed to date (Black and Rai, 1988). In mosquitoes, each rDNA transcriptional unit is composed of an external trans-cribed spacer, an $18 \mathrm{~S}$ subunit, an internal transcribed spacer 1 (ITS1), a 5.8S subunit, an ITS2, and a $28 \mathrm{~S}$ subunit. The rDNA units within an array are linked to each other by an intergenic spacer (IGS). The transcribed spacers are thought to contain conserved structures important in forming the mature ribosomal amplicon (Wesson et al, 1992; Paskewitz et al, 1993). The rDNA sequence is a valuable source of information because the functional regions that produce the ribosomes are highly conserved but the transcribed and nontranscribed spacers have the high interspecific and low intraspecific variability, making them useful for explaining relationships of recently diverged species and also useful as a basis for polymerase chain reaction (PCR) identification of morphologically similar species. As such, ITS1and ITS2 were used extensively in phylogenetic reconstruction of closely related and cryptic species complexes, as well as in the development of diagnostic species-specific PCR-based markers. However, because PCR can amplify all sequences of ITS present within the genome, variation among ITS sequences within individuals or species could result in accurate phylogenies and markers for species diagnostics. Consequently, the identifying and quantifying levels of intragenomic and intraspecific variations among ITS sequences are of real importance (Li and Wilkerson, 2007).

The aim of this study was to examine ITS2-rDNA sequences from multiple individuals of An. pharoensis and aligned with An. gambiae (Genbank, accession No. AY423072) to investigate the ITS2 genotype of the main malaria vector in Egypt and to evaluate its relation to Anopheles evolution.

\section{Materials and Methods}

This study of ITS2- rDNA variation conducted using individuals of $A n$. pharoensis colony from the Egyptian Medical Insect Institute, Dokki, Giza, Egypt and reared under the insectary conditions (temp. $27^{\circ} \mathrm{C}$ and $12 \mathrm{hr}$ light-dark) till adult stage and kept at $-80{ }^{\circ} \mathrm{C}$ until processing for DNA extraction. Genomic DNA isolated from single adult mosquito using Wizard Genomic DNA Purification Kit (Promega, cat \#A1620, USA).The Wizard Genomic DNA purification Kit (Promega) used with some modifications described by (Vidigal et al, 2000; Wassim, 2005). The DNA concentration determined using UV spectrophotometer at a wave length $=260 \mathrm{~nm}$ (Sambrook, 1989). The ITS2 region of rDNA was amplified using 5.8S primer (GTGGATCCTGTGGAACTGCAGGACA CATG) and 28S primer (GTG TCGACATGCTTAAATTTAGGGGGTA) (Wesson et al, 1992). Genomic DNA of single mosquito used for each reaction and PCR amplification, performed in $25 \mu 1$ using the following profile $5 \mathrm{~min}$ at $93^{\circ} \mathrm{C}$, 35 cycles $\left(1 \mathrm{~min}\right.$ at $93^{\circ} \mathrm{C}, 2 \mathrm{~min}$ at $58^{\circ} \mathrm{C}$, $1 \mathrm{~min}$ at $72^{\circ} \mathrm{C}$ ), and $7 \mathrm{~min}$ at $72^{\circ} \mathrm{C}$. The PCR products checked on $2 \%$ agarose gel electrophoresis with the ethedium bromide. The amplicons were excised and purified for sequencing analysis using QUIA quick gel 
extraction kit (Promega). The ampicons were directly sequenced and aligned to the sequence of ITS2-rDNA of An. gambiae available in Genbnak (Aceesion no. AY423072) and published by Garros et al. (2005) using (http://www. ebi.ac.uk /Tools/ msa/clustalw2/) then the expected secondary structure and free energy have been obtained.

\section{Results}

The ITS2 is referred to as the extent region between the $5.8 \mathrm{~S}$ and $28 \mathrm{~S}$ genes (coding regions) of rDNA from indivi-duals for the examined species (Figure 1). Using the above primers, we were able to identify the length of ITS2-rDNA of An. pharoensis (411) bp (Fig. 2), and study the genotype in comparison with An. gambiae (Garros, 2005).The length difference of ITS2-rDNA was $81 \mathrm{bp}$ between the two species. The sequence alignment of ITS2-rDNA of $A n$. pharoensis and An. gambiae showed rate of point mutations between the two species occurred at different variable nucleotide positions. There were 61 indels account-ing for both; (30 insertions and 21 deletions) and base substitutions (61 Transitions/103 Transversions). The GC content of the ITS2 reported here $(53 \%)$ is consistent with spacer base composi-tion in Anopheles species. The similarity between the two species was $52 \%$ and genetic distance was 0.46

ITS2-rDNA of An. pharoensis contained sequences tandem repeated, where an array of repeats located in different locations. Each repeat sequence was 3-4bp long. Some of the repeat are more common than others, the most common simple repeats were the trinucleotides; CGC, GCC, CTG, GGG, CGT, GCG, GGC, GCT, GTG, CTC, CGG and the tetranucleotides were CTCG, CTGC and GTGG (Tab. 1) less frequenting. The examination of the frequency of base composition of these repeats in $A n$. pharoensis showed that $41 \%$ of these repeats involved guanine followed by cytosine $33 \%$ and thymine $15 \%$. These repeats may under- line the relatively large degree of the sequence divergence between the Anopheline species. The genetic distance separating the two species was 0.46

The secondary structure of rDNA-ITS2 was predicted by MFOLD (Zuker et al, 1999). The minimum free energies were 192.60 to -195.32 kilocalories for $A n$. pharoensis. The structures of the two variants of An. pharoensis have the significantly high energy and lower stability. The stem and loop were clear in the presumptiveITS2 (Fritz et al, 1994) as in (Fig. 3). An. pharoensis had not identical base pairing between certain regions where sequence homology had already been differed. Most of the inter specific variability observed occurred outside the domains of the specific sequence homology of coding regions. The inter specific variability added to stem length, maintained a stem base pair, or occurred in a loop or other region of unpaired sequence.

\section{Discussion}

Unambiguous identification of Anophe-les malaria vector species is essential for the study of an array of factors that affect control and disease transmission. The phenomenon of lack of correspondence between the morphological similarity and similarity in the bionomics, vector potential, or insecticide resistance is well documented in the Anopheles mosquitoes (White, 1996). There is a growing body of evidence to suggest that within Anopheline taxa, genetic variation can be related to the adaptation of mosquito species to their environment (Coluzzi et al, 1979). An. pharoensis prefers the fresh water habitats (Marglit and Tahori, 1973; El Said et al, 1983; Mikhail et al, 2009) in Egypt. The genotypes have been associated with different ecotypes and geographical distribution, providing evidence of barriers to gene flow between populations of the species (Favia et al, 1997). The genotypes appear to relate to behavioral characteristics which have impor- 
tant implications in malaria trans-mission (Gakhar et al, 2013). The present results revealed that the length ITS2 of $A n$. pharoensis is $411 \mathrm{bp}$ and come quit together with the published sequences and unpublished Gen Bank submissions which show that most Anopheles ITS2s are ranged between 300 and 600 bp (Paskewitz et al, 1993; Fritz 1994; Wilkerson et al, 2004; Black et al, 2006; Oshaghi et al, 2013).

In the present study, direct sequence of ITS2-rDNA of An. pharoensis does not explain the intragenomic variations could not be expect the location of the rDNA arrays in An. pharoensis. The rDNA arrays are usually on chromosomes associated with sex determination. Kumar and Rai (1990) mapped dozens of species of mosquitoes and found rDNA loci on the autosomes of Culicine mosquitoes and on $\mathrm{X}$ and $\mathrm{Y}$ chromosomes of Anopheles. X and Y chromosomes in Anopheles are only partially homologous and $\mathrm{X}$ chromosome variants do occur (Rafael et al, 2003). Multiple rDNA locations are not unusual, for example, there are 5 in humans (Gonzalez and Sylvester, 2001) and at least 2 in Drosophila hydei (Hennig et al, 1975) and grasshoppers (White et al, 1982). Similar explanations were considered for other Anopheles mosquitoes by Onyabe and Conn (1999); Beebe et al. (2000) and Wilkerson et al. (2004). Physical mapping using in situ hybridization is needed to confirm the location of rDNA loci in the $A n$. pharoensis species (Sahel Sudanese form).

The mutation rates are higher than homogenization rates at ITS2 than other parts of the rDNA gene array, is probably very common (Harris and Crandall, 2000). Molecular events such as the insertions, deletions, duplication and an equal crossingover in sub repeat regions can generate spacer length heterogeneity. The results came quit to the above conclusion whereas the ITS2 sequence divergence between the two species has been maximal and occur at 61 indels (30insertions and 21 deletions).
The point mutations were 51 transitions and 103 transversions. Homogeneity theoretically decrease as mutation rate and the repeat number increase. The observation on the degree of homogenization among family members in a species can indicate at which levels of heterogeneity is gene-rated. Many of the genomic charac-teristics that have been described as conducive to faster rates of the homo-genization and fixation were more characteristics of Anopheline mosquetoes than they are of Aedine species (Levinson and Gutman, 1987)

The inter-specifically variable simple sequence repeats (SSRs) are found at low frequency in ITS2 of An. pharoensis. Sixty one indels in regions of SSRs and simple base repeat motifs account for most of the sequence variation observed and suggest their role as a major cause of divergence in the evolution of this spacer. The use of SSRs in Systematics has yet to be fully modeled. The process of SSM is more likely to be a major factor in the initial expansion of the short repeat motifs, which are subsequently pre-disposed to further expansion by an equal crossing over. A rapid rate of fixation of such mutations in tenderly repeated genes may subsequently distinguish closely and even distantly related invertebrates (Hancock et al, 1988). The observation of the addition SSRs at the tips of the secondary structure stems in $28 \mathrm{~S}$ variable regions. Slippage mechanism or nonhomologous exchange between chromosomes in these areas has resulted in the addition of SSRs with high self- similarity (Hassouna et al, 1984). These SSM are hot spots of slipped strand miss pairing events and these events in concert with unequal crossing-over can account for wide spread simple rDNA sequences.

The secondary structure model of $A n$. pharoensis is similar to the models proposed for Anopheles mosquitoes. The evidence that slippage synthesis is a major mechanism generating variability observed between related species may be due to some extent 
constrained by the secondary structure. Also, compensatory base change in the homologous regions and the hetero genoas highly variable areas, which tend toward GC rich simple sequences added to the length. This observation is based on evidences from secondary structure stem maintenance the complexity of the predicted structure was based on stems formed from base pairing between homologous domains (Levinson and Gutman, 1987). Under realistic rates of gene conversion, unequal exchange and transposition, fixation of mutant copy in a multigene family proceeds without a large variance at any given generation. This small variance is due to the much slower rates at which chromosomes distribute at each generation, so variance affected by many factors including this phenomena and the mechanism used to explain it have been termed the "concerted evolution" and "molecular drive" respectively (Dover, 1982).

\section{Conclusion}

The outcome results indicated that the comparison of ITS2-rDNA sequences and studying the secondary structures can be very useful for the analyzing of the uncharacterized vectors of malaria in Egypt. Phenomenon of lack of the correspondence between similarity in the bionomics, vector potential, or insecticide resistance is needed. Only by knowing the genetic identity of the vector under investigation can this particular part of an experimental design be accounted for furthermore, the subsequent studies or control efforts.

Next research should be focused on the use of molecular markers to identify other Anopheles mosquitoes in Egypt and the neighboring countries.

\section{References}

Beebe, NW, Cooper, RD, Foley, DH, Ellis, JT, 2000: Populations of the south-west Pacific malaria vector Anopheles farauti s.s. revealed by ribosomal DNA transcribed spacer polymerphisms. Heredity 84:244-53.
Black, WB, Rai KS, 1988: Genome evolution in mosquitoes: Interspecific and intra-specific variation in repetitive DNA amounts and organization. Genet. Res. 51:185-96.

Black, W, Brelsfoard, CL, Fritz, GN, Rodriguez, R, 2006: Sequence analysis of the rDNA internal transcribed spacer 2 and polymerase chain reaction identification of Anopheles fluminensis (Diptera: Culicidae: Anopheles) in Bolivia. J. Med. Entomol. 43, 3:460-6.

Bresfoard, C, McLain, DK, Rai, KS, 1989: Patterns of variation in the rRNAcistron within and among world populations of a mosquito Aedes albopictus (Skuse). Genetics 121:539-50.

Coluzzi, MA, Sabatini, V, Petrarca, MA, Di, D, 1979: Chromosomal differentiation and adaptation to human environment in the Anopheles gambiae complex. Trans. R. Soc. Trop. Med. Hyg. 73:483-97.

Dover, G, 1982: Molecular drive: a cohesive mode of species evolution. Nature 299, 5879: 111-7.

El Bahnasawy, MM, Dabbous, HK, Morsy, TA, 2010: Imported malaria as a threat to Egypt. J. Egypt. Soc. Parasitol. 40, 3:773-88.

El-Bahnasawy, MM, Saleh, NM, Khalil, M F, Morsy, TA, 2011: The impact of three anopheline mosquito species in Toshka, on the introduction of chloroquine resistant $P$. falciparum to Egypt. J. Egypt. Soc. Parasitol. 41, 3: 573-92.

El Said, S, Farid, H, 1982: Experimental transmission of Plasmodium vivax by Anopheles multicolor under the laboratory conditions and the effect of temperature on the sporogonic conditions and sporogonic cycle. J. Egypt. Pub. Hlth. Assoc. 57:512-40.

El Said, S, El Sawaf, B, Gebril, M, Kena- wy, MA, Azzab, BA, 1983: Bionomics of Anopheles in the Fayium Governorate, Egypt in relation to transmission and control of Malaria. J. Egypt. Pub. Hlth. Assoc. 58, 3/4: 12-9.

Favia, G, Della Torre, A, Yoko, BM, Branfrancotti, A, Sagnon, NF, et al, 1997: Molecular Identification of sympatric chromosomal forms of Anopheles gambiae and further evidence of their reproductive isolation. Insect Mol. Biol. 6, 4:377-83.

Fritz, GN, Conn, J, Cockburn, A, Seaw-right, J, 1994: Sequence analysis of the rDNA internal transcribed spacer 2 of populations of Anopheles nunestovari (Diptera: Culicidae). Mol. Biol. Evol. 11: 406-16. 
Gakhar, SK, Sharma, R, Sharma A, 2013: Population genetic Liston (Diptera: Culicidae) structure of malaria vector Anopheles stephensi. Indian J. Exp. Biol. 51:273-9.

Garros, C, Harbachin, SRE, Manguin, S, 2005: Morphological assessment and molecular phylogenetics of the funestus and Minimus Groups of (Cellia). J. Med. Entomol. 42:522536.

Gerbi, SA, 1985: Evolution of ribosomal. In: RJ, Mac-Intyre (ed.), Molecular Evolutionary Genetics, Plenum, New York.

Gonzalez, IL, Sylvester, JE, 2001: Human rDNA: Evolutionary patterns within the genes and tandem arrays derived from multiple chromosomes. Genomics 73:255-63.

Hancock, JM, Tautz, D, Dover, GA, 1988: Evolution of the secondary structures and compensatory mutations of the ribosomal RNAs of Drosophila melanogaster. Mol. Biol. Evol. Evol. 5:393-414.

Harris, DJ, Crandall, KA, 2000: Intra-genomic variation within ITS1 and ITS2 of fresh water crayfishes (Decapoda: Cambaridae): implications for phylogenetic and microsatellite studies. Mol. Biol. Evol. 17: 284-91.

Hassouna, NB, Michot, BA, Chellerie, JP, 1984: The complete nucleotide sequence of mouse 28S rRNA gene: Implications for the process of size increase of the large subunit rRNA in higher eukaryotes. Nucl. Acids Res. 12:3563-83.

Hennig, W, Link, B, Leoncini, O, 1975: The location of nucleolus organizer regions in Drosophila hydei. Chromosoma 51:57-63.

Kumar, A, Rai, KS, 1990a: Chromosomal localization and copy number of $18 \mathrm{~S}+28 \mathrm{~S}$ ribosomal RNA genes in Evolution are diver-se mousquites (Diptera: Culicidae). Heredit-ies 113:227-89.

Levinson, G, Gutman, GA, 1987: Slipped strand mispairing: a major mechanism for DNA sequence evolution. Mol. Biol. Evol. 4, 3:20321.

Li, C, Wilkerson, RC, 2007: IntragenomicroDNA ITS2 variation in the neotropical Anopheles (Nyssorhynchus) albitarsis complex (Diptera: Culicidae). J. Heredity 98, 1: 51-9.

Marglit, J, Tahori, AS, 1973: The mosquito fauna of Sinai. J. Med. Entomol.10:89-96.

Miles, SJ, Green, CA, Hunt, RH, 1983: Genetic observations on the taxon Anopheles
(Cellia) pharoensis Theobald (Diptera: Culicidae). J. Trop. Med. Hyg. 86:153-7.

Mikhail, MW, Al-Bursheed, KM, Abd ElHalim, AS, Morsy, TA, 2009: Studies on mosquito borne diseases in Egypt and Qatar. J. Egypt. Soc. Parasitol. 39, 3:745-56.

Mouchet, J, Carnevale, P, Manguin, S, 2008: Biodiversity of Malaria in the World. Esther, UK: John Libbey Eurotext.

Onyabe, DY, Conn, JE, 1999: Intragenomic heterogeneity of a ribosomal. DNA spacer (ITS2) varies regionally in the neo-tropical malaria vector Anopheles nuneztovari (Dip-tera: Culicidae). Insect Mol. Biol. 8:435-42.

Oshaghi, MA, Sedaghat, MM, Vatandoost, H, 2003: Molecular characterization of the Anopheles maculipennis complex in the Isla-mic Republic of Iran. East. Mediterr. Hlth. J. 9, 4:659-66.

Paskewitz, SM, Wesson, DM, Collins, FH, 1993: The internal transcribed spacers of ribosomal DNA in +ve members of the Anopheles gambiae species complex. Ins. Mol. Biol. 2:247-57.

Rafael, MS, Tadei, WP, Pimentel, SM, 2003: Location of Ribosomal Genes in the Chromosomes of Anopheles darlingi and Anopheles nuneztovari (Diptera, Culicidae) from the Brazilian Amazon. Mem. Inst. Oswaldo Cruz, Rio de Janeiro 98, 5:629-35.

Sambrook, J, Fritsch, EF, Maniatis, T, 1989: Molecular cloning: A laboratory Manual, $2^{\text {nd }}$ ed. Cold Spring Harbor Labora-tory, Cold Spring Harbor, NY.

Tautz, D, Hancock, JM, Webb, DA, Tautz, C, Dover, GA, 1988: Complete sequences of the rDNA genes of Drosophila melanogaster. Mol. Biol. Evol. 5:366-76.

Vidigal, TH, Kissinger, JC, Caldeira, RL, Pires, EC, Monteiro, E, et al, 2000: Phylogentic relationship among Brazilian Biomphalaria species (Mollusca: Planorbidae) based upon analysis of ribosomal ITS2 sequences. Parasitol. 121, 6:611-20.

Wassim, NM, 2005: Sequence Analysis of the Second Internal Transcribed spacer region of Ribosomal DN (ITS2-rDNA) of Anopheles multicolor (Cambouliu) populations from different isolated geographical areas of Egypt. Egypt. Ger. Soc. Zool. 46, E:161-72, Cairo, Egypt.

Wesson, DM, Porter, CH, Collins, FH, 1992: Sequence and secondary structure comparisons 
of ITS rDNA in mosquitoes (Diptera: Culicidae). Mol. Phylogenet. Evol. 1:253-69.

White, NJ, 1996: The treatment of malaria. Engl. J. Med. 335:800-6.

White, MJ, Dennis, ES, Honeycutt, RL, Contreras, N, 1982: Cytogenetics of the parthenogenic grasshopper Warramabavirgo and its bisexual relatives. IX. The ribosomal RNA cistrons. Chromosoma 85:181-99.

Wilkerson, RC, Reinert, J, Cong, LI, 2004: Ribosomal DNA ITS2 sequence differentiate six species in the Anopheles crucians complex (Diptera: Culicidae). J. Med. Entomol. 41, 3: 391-400.
Zahar, AR, 1975: Review of the ecology of malaria vectors in the WHO eastern Mediterranean Region. Bull WHO. 50:427-40.

Zahar, AR, 1989: Vector Bionomics in the Epidemiology and Control of Malaria. Part IIWHO European region and WHO Eastern Mediterranean Region. Vol. II. Section II. VBC/90, 2:226-32.

Zuker, M, Mathews, DH, Turner, DH, 1999: Algorithms and thermodynamics for RNA secondary structure prediction: A practical guide in RNA biochemistry and biotechnology. In: Barciszewski, J, Clark, BFC, editors. NATOASI Series. Dordrecht (Netherland): Kluwer Academic Publishers.

Table 1: simple sequence Repeats (SSRs) in ITS2-rDNA of An. pharoensis and An. gambiae

\begin{tabular}{|c|c|c|}
\hline SSRs & An. gambiae & An. pharoensis \\
\hline CGC & 3 & 11 \\
\hline GGG & 7 & 2 \\
\hline GCC & 6 & 7 \\
\hline CTG & 4 & 14 \\
\hline GTG & 8 & 4 \\
\hline CTC & 3 & 6 \\
\hline GGC & 12 & 8 \\
\hline GCG & 7 & 9 \\
\hline CGT & 10 & 5 \\
\hline CGG & 5 & 4 \\
\hline GCT & 9 & 11 \\
\hline GTGG & 4 & 3 \\
\hline CTCG & 4 & 6 \\
\hline CGCG & 2 & 3 \\
\hline
\end{tabular}

Fig.1: PCR amplifications of ITS2- rDNA of An.pharoensis (lane 2, 3) \& PEGM-DNA marker (lane 1)

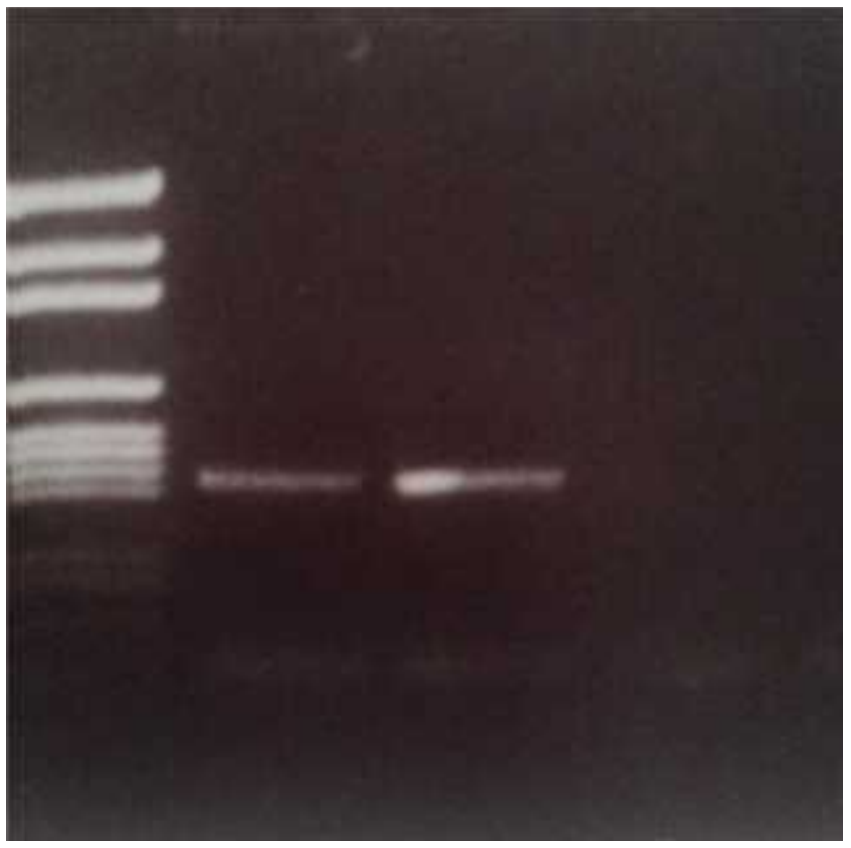


An.pharonsis An. gambiae

An.pharonsis An. gambiae

An.pharonsis An. gambiae

An.pharonsis An. gambiae

An.pharonsis An. gambiae

An.pharonsis An. gambiae

An.pharonsis An. gambiae

An.pharonsis An. gambiae

An.pharonsis An. gambiae

-------TGGCTTT-----CCGCCAGCTGCAGGAAAGTTT------------- ${ }^{\text {TCACCAC } 35}$ GCGCATCGGACGTTTAATCCCGACCGATGCACACATTCTTGAGTGCCTACTAATTACCAA 60

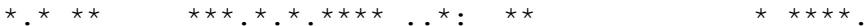

G--CTCAT-------CCGCTCGGGCCTGCTGCCAG---ACCCTGGTGTGCCACATTCGC 82 AGTCTCATTTAGTTAACTACAGTGGCCGTCCGCGAAGGTGCCCGGGT---CATCCGACGC 117

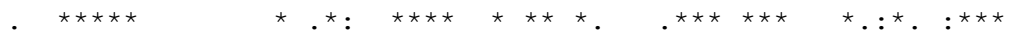

ACTGTGACTGTCTGCTGTGTATACT-CCTCGAATTGTCCGC-TCT-CGCTTTC-CGGTCA 138 ACTG-GGCGGTC-GCTGTGCATAATGACGTGCTTGGTCCCCGTCTGCGGGTCCTCGGGCG 175

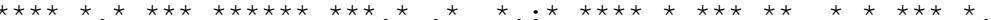

TATAGCGCAATTCCATCTCGCGACCTTTGACCGGAATGTGCCG-AATGCTGGCATCACAT 197 TTGAAAGTGG-ACACTCTCGAG-CGTATGTTGGATGCGTTTCGTGTTGGTGGTGTTTGAT 233

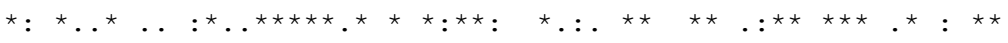

GCGAATCATCTGTGGCTGACTGCCTTGGTGCACTGT-----CTCTGGCGTCGTCGGGGGC 252 GCGTAGGGCTTGTGG-TGTGTGTCAAGCCGCATGGTTCGAACTAATGCTACGTCGTTCCC 292

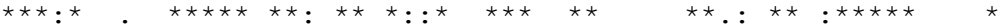

TTTGGATCGTTCGGGTCAAAGGTCGCTAGTATGGAATTGCCATTGATGACAGGAAAGGCA 312 GATGG--CCACCGGCAGTCTACTCTCCAGGCTAAAGTCGGCT----CGTCTAGGGATTCG 346

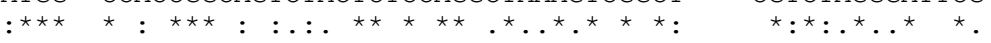

TTGAGCTAACTTGATGGATTAAACAGCGTCCCATTGTCAGTTTGTGGCACACAAACATTG 372 GAAAGCTAAGTCGCTGTAACTCATGTGGGCCCATACACGG--CGTTGCGCTACCACGCT- 403

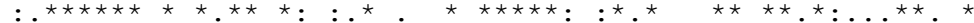

AACGCTGGCCCTGACATATTTAGATTCTCGCAAAACATCTCGCATCGGAAAACACCATTT 432 -AAGTTAGCCCT-ACATAT-----ACAAGCATCAACCCACGGCACGGGCGTAGCTGTAA 455

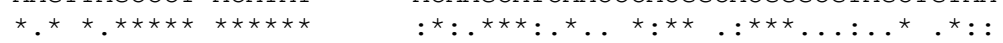

TGCCCACAACGCG-------CGTGCAG-CCCCATGGGAAAA----- 465 TACTTACGTCTCGGTTATACCACGTAGGCCTCAAGTGATGTGTGAC 501

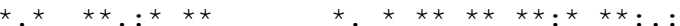

Fig. 2: Sequence alignment of the ITS2-rDNA and flanking 5.8S and 28S genes of An. pharoensis and An. gambiae. Asterisks indicate identical nucleotide positions. Alignment generated by CLUSTALW 2

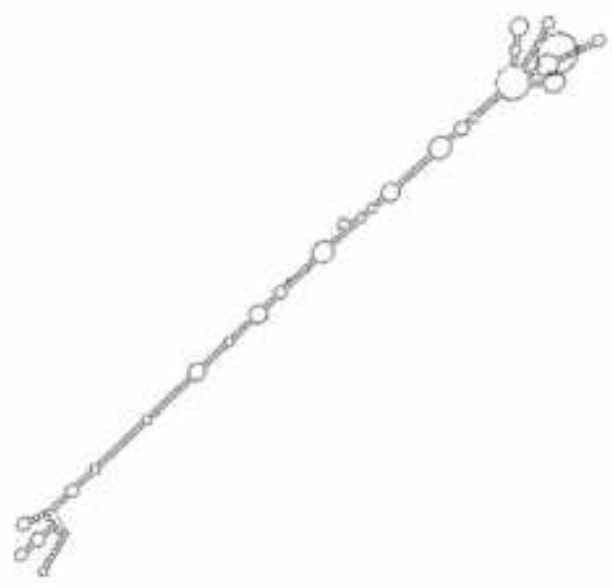

Fig.3: Secondary Structure of ITS2-rDNA of An. pharoensis 